\title{
1 Cardiotoxin-induced skeletal muscle injury elicits profound changes in anabolic 2 and stress signaling, and muscle fiber type composition
}

\author{
3 Sebastiaan Dalle ${ }^{\mathrm{a}}$, Charlotte Hiroux ${ }^{\mathrm{a}}$, Chiel Pofféa ${ }^{\mathrm{a}}$, Monique Ramaekers ${ }^{\mathrm{a}}$, Louise Deldicque ${ }^{\mathrm{b}}$, Katrien Koppo ${ }^{\mathrm{a}^{*}}$ \\ $4 \quad{ }^{a}$ Exercise Physiology Research Group, Department of Movement Sciences, KU Leuven, Tervuursevest 101, 3001 Leuven, \\ 5 Belgium
}

6 'bnstitute of Neuroscience, Université Catholique de Louvain, Place Pierre de Coubertin 1, 1348 Louvain-la-Neuve, 7 Belgium

8 Sebastiaan Dalle: https://orcid.org/0000-0001-5734-3993

9 Charlotte Hiroux: https://orcid.org/0000-0001-8345-0119

10 Chiel Poffé: https://orcid.org/0000-0002-8085-3075

11 Louise Deldicque: https://orcid.org/0000-0003-3393-5278

12 Katrien Koppo: https://orcid.org/0000-0002-6022-1097

13 * Corresponding author:

14 Katrien.koppo@kuleuven.be

\section{Abstract}

16 To improve muscle healing upon injury, it is of importance to understand the interplay of key signaling pathways during 17 muscle regeneration. To study this, mice were injected with cardiotoxin (CTX) or PBS in the Tibialis Anterior muscle and 18 were sacrificed 2, 5 and 12 days upon injection. The time points represent different phases of the regeneration process, i.e. 19 destruction, repair and remodeling, respectively. Two days upon CTX-injection, p-mTORC1 signaling and stress markers 20 such as BiP and p-ERK1/2 were upregulated. Phospho-ERK1/2 and p-mTORC1 peaked at d5, while BiP expression 21 decreased towards PBS levels. Phospho-FOXO decreased two and five days following CTX-injection, indicative of an increase in catabolic signaling. Furthermore, CTX-injection induced a shift in the fiber type composition, characterized by an initial loss in type IIa fibers at $\mathrm{d} 2$ and at $\mathrm{d} 5$. At $\mathrm{d} 5$, new type IIb fibers appeared, whereas type IIa fibers were recovered at d12. To conclude, CTX-injection severely affected key modulators of muscle metabolism and histology. These data provide useful information for the development of strategies that aim to improve muscle molecular signaling and thereby

26 recovery.

27 Keywords: cardiotoxin, inflammation, muscle injury, muscle metabolism, muscle regeneration 


\section{Declarations}

29 Funding: This research did not receive any specific grant from funding agencies in the public, commercial, or

30 not-for-profit sectors.

31 Conflicts of interest/Competing interests: The authors declare no conflicts of interest.

32 Ethics approval: All procedures performed in studies involving animals were in accordance with the ethical standards of 33 the institution or practice at which the studies were conducted (KU Leuven Animal Ethics Committee; P168/2016)

34 Consent to participate: N.A.

35 Consent for publication: All authors agreed with the content and gave explicit consent to submit. All authors obtained

36 consent from the responsible authorities at the institute/organization where the work has been carried out, before the work 37 is submitted.

38 Availability of data and material (data transparency): Data are presented in figures and tables.

39 Code availability: N.A.

40 Authors' contributions: Conception and design of the study: SD and KK. Data collection and analysis: SD, MR, CP and

$41 \mathrm{CH}$. Analysis and interpretation of the data: SD, LD and KK. Manuscript drafting: SD and KK. 
43 Muscle injuries are a widely occurring event. Improper muscle regeneration might have long-term consequences, e.g. a decreased functional capacity. Therefore, many studies focus on strategies to improve the regeneration process. Since ethical and practical constrains limit investigating muscle injuries in humans, animal muscle injury models provide valuable insights in the regeneration process. The main advantages of these models, when compared to injuries in humans, involve a fast regeneration process (most of the muscle histology is repaired within 2 weeks), standardization of injury and feasibility to apply complex interventions, eventually resulting in a better understanding of the recovery process on microand macro-molecular level. Animal muscle injury models can roughly be categorized in mechanical-induced injuries, such as contusion injury, muscle overload, freeze injury and pressure injury, and chemical-induced injuries, e.g. by injecting myotoxic substances in skeletal muscle tissue, such as cardiotoxin (CTX), notexin, buvicain or glycerol(Mahdy 2018).

Upon muscle injury, the regeneration process exhibits distinct sequential phases, i.e. a destruction, repair and remodeling phase(Järvinen et al. 2005). The timing of each phase and of the up- and downregulation of particular pathways involved in the regeneration varies amongst mechanical- and chemical-induced muscle damage models(Lefaucheur and Bille 1995; Fink et al. 2003; Czerwinska et al. 2012), but also between different chemical-induced damage models(Mahdy et al. 2015). This has led to a welter of heterogeneous information on muscle regeneration, depending on the applied injury model, the type of injured muscle, the biochemical targets, parameters of muscle functionality, and the time point(s) within the regeneration process. Hence, it is not straightforward for researchers to predetermine and compare relevant outcome parameters with results from other studies, or to select an appropriate time point in the regeneration process for given outcome variables.

61

The present study sheds light on the complex multiplicity of information by describing both muscle histological changes and alterations in key molecular pathways at several time points during the regeneration process. Muscle injury was evoked by an acute CTX-injection. This model is highly eligible to study muscle recovery as the regeneration process occurs relatively efficient due to a proper blood supply and the preservation of basal lamina and microvasculature(Harris 2003; Czerwinska et al. 2012). CTX-injection is also less harmful for the animal compared to mechanical injuries(Couteaux et al. 1988). The CTX-induced regeneration model establishes similarities with diverse myopathies and might therefore serve as a tool to study these pathologies(Pessina et al. 2014; Sunitha et al. 2016). Besides, fundamental insights in muscle regeneration (both on molecular and histological level) contribute to our understanding of muscle injury and eventually to the development of novel strategies that might improve muscle healing upon injuries.

Recent advances in techniques, such as microarrays, allow to study a very broad range of targets following CTX injury(Goetsch et al. 2003; Yan et al. 2003). Despite the advantage of generating big data, this approach also encounters some limitations: i) mRNA expression is less physiologically relevant than posttranslational responses (e.g. phosphorylated proteins), ii) often small sample sizes ( $n=3$ /group), iii) hypothesis-free research which is not straightforward to interpret and to directly implement in physiological research. Therefore, we aim to study the (phosphorylated) protein levels of central targets that are expected to play a significant and distinct role in muscle regeneration and of which the changes during the regeneration process have not been described yet. We selected several stress markers (BiP, ERK1/2 and p38 MAPK and FOXO1/3a signaling) and anabolic markers (mTORC1 signaling pathway) which provide useful information about the response of the muscle tissue to injury, as well as the fiber type composition which is a key feature of the muscle phenotype.

\section{$80 \quad$ Materials and Methods}

Animal use - All procedures performed in studies involving animals were in accordance with the ethical standards of the institution or practice at which the studies were conducted (KU Leuven Animal Ethics Committee; P168/2016). Forty-eight young $(10 \mathrm{w})$, male C57BL/6 mice were purchased from Janvier Labs (France). In half of the group $(\mathrm{n}=24)$, the middle portion of the m. Tibialis Anterior (TA) of both hindlimbs was injected with sterile CTX (10 $\mu$ M; L8102, Latoxan, France) dissolved in sterile $150 \mu 1$ phosphate-buffered saline (PBS), while the other half was injected with $150 \mu 1$ sterile PBS $(n=24)$ with a BD microfine 300- $\mu \mathrm{L}$ U-100 insulin syringe. Prior to TA injection, mice were anaesthetized by intraperitoneal (IP) injection of $10 \mu \mathrm{L} \cdot \mathrm{g}^{-1}$ body mass (BM) of saline solution containing $5 \%$ Rompun $\left(100 \mathrm{mg} \cdot \mathrm{mL}^{-1}\right.$ xylazine) and $10 \%$ Nimatek (100mg $\cdot \mathrm{mL}^{-1}$ ketamine). In each condition, 8 mice were sacrificed respectively 2,5 and 12 days following CTX or PBS injection. Mice abstained from food 2-3h prior to their sacrifice. TA muscles were surgically removed and weighed. One TA muscle was snap frozen in liquid nitrogen and stored at $-80^{\circ} \mathrm{C}$ for later protein extraction, whereas the other TA muscle was frozen in liquid nitrogen-cooled isopentane and later sectioned at a thickness of $7 \mu \mathrm{m}$ for histological analyses. 
Histological analyses - Muscle tissue embedded in tissue freezing medium (Leica Biosystems, Germany) was frozen in liquid nitrogen-cooled isopentane. Serial 7- $\mu \mathrm{m}$-thick cryosections were cut with a cryostat (Leica Biosystems CM1850, Germany) at $-20^{\circ} \mathrm{C}$. Prior to the histological analyses, cryosections were thawed at room temperature, washed with PBS and fixed with $4 \%$ paraformaldehyde. To permit qualitative analysis of morphological alternations, cryosections were stained with haematoxylin and eosin (H\&E) (Sigma Aldrich, USA), and with fibronectin (FN) + Hoechst. For the FN staining, cryosections were blocked for 30min in PBS containing 5\% bovine serum albumin (BSA). Next, cryosections were incubated for $1 \mathrm{~h}$ at $37^{\circ} \mathrm{C}$ in a humid chamber with anti-FN antibody (Abcam ab2413; 1:200 dissolved in 1\% BSA in PBS). After washing PBS, cryosections were incubated for $1 \mathrm{~h}$ at room temperature with the conjugated donkey anti-rabbit IgG secondary antibody Alexa-488 (1:500), washed with PBS, incubated with Hoechst $\left(1 \mu \mathrm{g} \cdot \mathrm{mL}^{-1}\right)$ for $15 \mathrm{~min}$ at room temperature. To obtain fiber type composition, cryosections were blocked for $2 \mathrm{~h}$ in PBS containing $1 \%$ bovine serum albumin (BSA). Following permeabilisation in PBS (1\% BSA $+0.2 \%$ triton) for $15 \mathrm{~min}$, cryosections were incubated overnight at $4^{\circ} \mathrm{C}$ in a humid chamber with the following primary antibodies (Developmental Studies Hybridoma Bank, USA): BA-F8 (1:400, myosin heavy chain (MyHC) I), SC-71 (1:100, MyHC IIa), BF-F3 (1:300, MyHC IIb) and L9393 (1:500, Laminin, Sigma Aldrich, USA) dissolved in PBS. After washing in PBS, cryosections were incubated for $1 \mathrm{~h}$ at room temperature with the following conjugated secondary antibodies (Life Technologies, USA): goat anti-mouse Alexa488 IgG2 (1:300, MyHC I), goat anti-mouse Alexa-350 IgG1 (1:300, MyHc IIa), goat anti-mouse Alexa-594 IgM (1:300, MyHC IIb), donkey anti-rabbit Alexa-488 IgG (1:600, laminin). All immunofluorescence sections were mounted with Dako fluorescence mounting medium (Dako, S3023) and H\&E-stained sections were covered with DPX mountant for histology (Sigma 06522).

110

111

112

113

114

115

Slides were visualized by fluorescence microscopy (Nikon E1000, Germany). The epifluorescence signal was recorded with FITC, DAPI and Texas Red excitation filters for visualization of MyHC I and cell membranes, MyHC IIa, and MyHC IIb, respectively, and with FITC and DAPI for visualization of FN and Hoechst, respectively. Muscle fibers of the full cryosection were classified as type I, type II, type IIb or unstained. Images of the slides were analyzed with ImageJ software (version 1.41, National Institutes of Health, USA).

Protein extraction - One total TA muscle was manually homogenized with a mortar, dissolved in ice-cold lysis buffer [1:10, w/v; 50mM Tris-HCl, pH 7.0; 270mM sucrose; 5mM EGTA; 1mM EDTA; 1mM sodium orthovanadate; 50mM glycerophosphate; $5 \mathrm{mM}$ sodium pyrophosphate; $50 \mathrm{mM}$ sodium fluoride; $1 \mathrm{mM}$ dithiothreitol; $0.1 \%$ Triton X-100; and a complete protease inhibitor tablet (Roche Applied Science, Belgium)] and centrifuged at $10000 \mathrm{~g}\left(25 \mathrm{~min}, 4^{\circ} \mathrm{C}\right)$. Next, the supernatant was stored at $-80^{\circ} \mathrm{C}$. The protein concentration was assessed with the DC protein assay kit applying a BSA protein standard (Bio-Rad Laboratories, Belgium). Lysis buffer was added to equalize protein concentrations. Eventually, laemmli ( $20 \%$ of the total volume) was added to obtain muscle lysates.

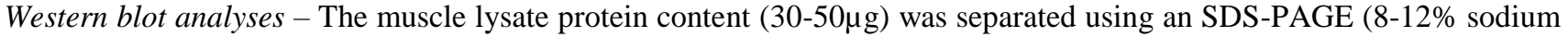
acrylamide) and were transferred to polyvinylidene difluoride membranes, which were next blocked in tris-buffered saline tween-20 (TBS-T) with 5\% BSA for 1h and incubated with the primary antibody, dissolved 1:200-1:10000 in 5\% BSA in TBS-T, at $4^{\circ} \mathrm{C}$ overnight: phospho-Akt ${ }^{\text {Ser473 }}$ (CST5171; 1:1000), phospho-mTOR ${ }^{\text {Ser2448 }}$ (CST2971S; 1:1000), phosphoS6K1 ${ }^{\text {Thr389 }}$ (CST9206S; 1:1000), phospho-S6 Ribosomal Protein ${ }^{\text {Ser235/236 }}$ (CST2211; 1:1000), phosphoFoxO1 $^{\text {Thr24/FoxO3a }}{ }^{\text {Thr32 }}$ (CST9464S; 1:1000), phospho-p44/42 MAPK (Erk1/2) ${ }^{\text {Thr202/Tyr204 }}$ (CST9101; 1:1000), phospho-

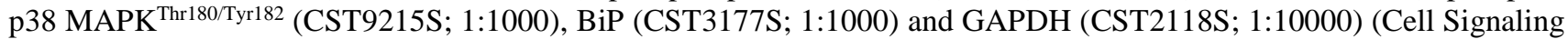
Technology, The Netherlands). Secondary anti-mouse (1:7000) and anti-rabbit (1:5000) antibodies conjugated to horseradish peroxidase were applied to detect target proteins. Next, protein bands were quantified with the GeneSnap software (Syngene, UK). Since the CTX-injection increased the total protein form (Suppl. Fig. 1), proteins were presented relative to the total protein loading stained with Ponceau (Suppl. Fig. 2).

Statistical analyses - All values are presented as means \pm SEM. Non-normal data were log transformed and a two-way ANOVA with Tukey post-hoc tests was performed to assess differences among conditions and times. Statistical significance was accepted for $\mathrm{p}<0.05$. All statistical analyses were performed with SPSS (Version 22.0.0.0; IBM Corp; USA).

\section{Results}

138 Muscle injury model (Table 1; Fig. 1; Fig. 2) - We analyzed the TA muscle 2, 5 and 12 days following PBS-injection to 139 check whether the injection per se affected the selected molecular targets and muscle regeneration. PBS-injection did not induce skeletal muscle injury and regeneration (Fig. 1). Also, there was no effect of PBS-injection on BM or TA mass (table 1). CTX-injection induced no changes in BM compared to PBS, i.e. $\triangle \mathrm{BM}$ (at pre-TA injection $v s$. at the day of sacrifice) was $+0.05 \pm 0.4 \mathrm{~g}$ in PBS at $\mathrm{d} 2$, and $-0.8 \pm 0.1 \mathrm{~g}$ at $\mathrm{d} 2(\mathrm{p}=0.38),-0.5 \pm 0.4 \mathrm{~g}$ at $\mathrm{d} 5(\mathrm{p}=0.69)$ and $+0.2 \pm 0.7 \mathrm{~g}$ at $\mathrm{d} 12$ $(\mathrm{p}=0.61$ ) in CTX (table 1). At $\mathrm{d} 5$, TA mass was significantly lower in CTX compared to PBS ( $<0.001$ ), while no 
differences were observed at $\mathrm{d} 2(\mathrm{p}=0.317)$ and $\mathrm{d} 12(\mathrm{p}=0.937)$. CTX-injection effectively induced muscle injury and regeneration, as the destruction, repair and remodeling phase were clearly distinguishable at $\mathrm{d} 2, \mathrm{~d} 5$ and $\mathrm{d} 12$. The early response upon CTX-induced muscle injury (i.e. d2) was characterized by infiltration of mononucleated cells (Fig.1 and Fig. 2) and interruption of the myofiber integrity (Fig.1). At the same time, FN, a glycoprotein present in the sarcolemma and connective tissue, was highly expressed in the damaged fibers, whereas FN was only expressed in the sarcolemma of PBSinjected fibers (Fig. 2). At d5, infiltrated cells remained present to a lesser extent and regenerating myofibers with centralized nuclei were present (Fig. 1). Furthermore, the FN expression was still present in some, but not all, fibers in damaged areas (Fig. 2). Infiltrated cells were present in the fibers that highly expressed FN, but not in undamaged fibers that only expressed FN in the sarcolemma (Fig. 2). Twelve days following CTX-injection, regenerating myofibers with centralized nuclei were enlarged compared to d5 (Fig. 1). The FN staining revealed that all fibers in regenerating areas expressed FN in the sarcolemma and not within the fibers (Fig. 2).

Shift in TA muscle fiber type (Fig. 3; Table 2) - As the amount of type I fibers was $<1 \%$ and remained unchanged following compared to PBS $(\mathrm{p}=0.008)$, whereas unstained (type IIx and immature) fibers and type IIb fibers were not significantly different $(\mathrm{p}=0.30 ; \mathrm{p}=0.20)$. At $\mathrm{d} 5$, the amount of type IIa fibers remained low $v s$. PBS $(\mathrm{p}=0.002)$ and the amount of unstained $(\mathrm{p}=0.004)$ and type IIb ( $\mathrm{p}=0.001)$ were significantly higher than PBS. At d12, the amount of the type IIa, IIb and unstained fibers returned to PBS values. The relative contribution of each fiber type was assessed by dividing its respective absolute amount by the total amount of fibers. Upon CTX-injection, the type IIa fibers decreased $\sim 11 \%$ at $\mathrm{d} 2 v s$. PBS ( $\mathrm{p}=0.011$ ), while unstained fibers were higher $(+20 \%$; $\mathrm{p}=0.008)$ and IIb fibers tended to be decreased $(-10 \% ; \mathrm{p}=0.08)$. Five days upon CTX-injection, type IIa fibers remained low $(-\sim 12 \%$; $<<0.001)$, whereas type IIb and unstained fibers did not differ from PBS. At d12, none of the fiber types differed from PBS.

Fiber type specific characteristics (Table 2) - Two days upon CTX-injection, the minimum Feret diameter of all fiber types were non-significantly decreased compared to PBS. At d5, unstained fibers had a significantly lower minimum Feret diameter in CTX $(21.9 \pm 1.3 \mu \mathrm{m}) v s$. PBS $(31.3 \pm 2.3 \mu \mathrm{m} ; \mathrm{p}=0.005)$. Accordingly, the diameter of type IIb fibers was lower five days following CTX-injection $(25.2 \pm 1.9 \mu \mathrm{m})$ compared to PBS $(43.8 \pm 2.1 \mu \mathrm{m} ; \mathrm{p}<0.001)$. Twelve days following injection, there was a trend towards a decreased minimum Feret diameter of type IIa fibers in CTX $(27.0 \pm 2.2 \mu \mathrm{m}) v s$. PBS $(33.5 \pm 4.2 \mu \mathrm{m} ; \mathrm{p}=0.075)$, and the diameter of unstained and type IIb fibers was significantly lower following CTX $(28.4 \pm 3.4 \mu \mathrm{m} v s .36 .5 \pm 2.2 \mu \mathrm{m} ; \mathrm{p}=0.012$ and $32.9 \pm 3.9 \mu \mathrm{m} v s .47 .6 \pm 4.6 \mu \mathrm{m} ; \mathrm{p}=0.003$, respectively).

Muscle stress response (Fig. 4) - Stress markers and catabolic markers were severely affected by the CTX-injection. All markers, except for p-p38 MAPK, exhibited a significant treatment effect $(\mathrm{p}<0.05)$. First, BiP protein expression increased at $\mathrm{d} 2$ (22-fold $v s$. PBS; $\mathrm{p}<0.001)$ and gradually returned towards PBS-levels 5 days (2-fold $v s$. PBS; $\mathrm{p}=0.216)$ and 12 days following CTX-injection ( $\mathrm{p}=0.788$ ) (Fig. 4a). Second, the p-ERK1/2 MAPKs were significantly increased at $\mathrm{d} 2$ ( 2-fold $v s$. PBS; $\mathrm{p}<0.05)$, peaked at $\mathrm{d} 5(\sim 3$-fold $v s$. PBS; $\mathrm{p}<0.001)$. The increment in $\mathrm{p}$-ERK1/2 at $\mathrm{d} 12$ was less pronounced $(1.7-$ fold $v s$. PBS; $\mathrm{p}=0.072$ for $\mathrm{p}$-ERK1 and $\mathrm{p}=0.163$ for $\mathrm{p}$-ERK2) (Fig. 4b, c). Phosho-p38 was increased at d2 ( 2-fold vs. PBS; $\mathrm{p}=0.038)$ and decreased at $\mathrm{d} 5$ (0.4-fold $v s . \mathrm{PBS} ; \mathrm{p}=0.004)$, and was non-significantly decreased 12 days upon CTX-injection (0.5-fold $v s$. PBS; $\mathrm{p}=0.221$ ) (Fig. 4d). Finally, p-FOXO1/3a expression was lower in the CTX-group at $\mathrm{d} 2$ (0.3-fold $v s$. PBS; $\mathrm{p}=0.002)$ and at $\mathrm{d} 5$ (0.4-fold $v s . \mathrm{PBS} ; \mathrm{p}=0.009)$, whereas the decreased expression at $\mathrm{d} 12$ was no longer significant (0.7-fold vs. PBS; $\mathrm{p}=0.302)$ (Fig. 4e).

182

183

Muscle anabolic response (Fig. 5) - All anabolic markers were significantly upregulated due to CTX compared to PBS injection ( $\mathrm{p}<0.05$ ). Phospho-Akt expression was increased 2 days (3-fold $v s$. PBS; $\mathrm{p}=0.02$ ) and peaked 5 days following CTX-injection (4-fold $v s$. PBS; $\mathrm{p}<0.001$ ), while the increase was no longer significant at d12 (2-fold; $\mathrm{p}=0.218$ ) (Fig. 5a). In contrast to $\mathrm{p}-\mathrm{Akt}$, the increased $\mathrm{p}-\mathrm{mTOR}$ expression 2 days upon CTX injection was not significantly different from PBS (1.4-fold vs. PBS; $\mathrm{p}=0.138)$. At d5, p-mTOR expression peaked ( 3-fold $v s$. PBS; $\mathrm{p}<0.001)$ whereas at $\mathrm{d} 12$, there was no different expression compared to PBS (1-fold vs. PBS, p=0.969)(Fig. 5b). Phospho-S6K1 and p-S6rp were significantly increased at $\mathrm{d} 2$ (8- and 4-fold $v s$. PBS; $\mathrm{p}=0.004$ and $\mathrm{p}=0.001$, respectively) and at $\mathrm{d} 5$ (4-fold $v s$. PBS; $\mathrm{p}=0.089$ and $\mathrm{p}<0.001$, respectively) (Fig. 5c, d). At d12, both parameters did not significantly differ between PBS and CTX.

\section{Discussion}

191 This is the first study in which the posttranslational patterns of distinct key molecular pathways (mTORC1, MAPK, FOXO) 192 and muscle histological changes are simultaneously displayed during muscle regeneration following CTX-induced injury. 193 Two days upon injury, the stress response was characterized by an upregulation of BiP and a decreased amount of type IIa 194 fibers. Next, at d5, unstained (immature and type IIx) and type IIb fibers were higher in CTX vs. PBS, with a concomitant 
upregulation of mTORC1 and ERK1/2 signaling. Twelve days following the CTX-injection, the muscle phenotype was recovered towards the phenotype of a 'healthy' PBS-injected muscle.

197 Fiber type shift - We demonstrated that the slower type IIa fibers were more vulnerable to CTX-injection and that the formation of primarily fast muscle fibers preceded the slow fibers in the TA, i.e. slow type IIa fibers were decreased at $\mathrm{d} 2$ and d5 but not at d12 in CTX vs. PBS, whereas fast IIb fibers were higher at d5 compared to PBS (Fig. 3). These observations are in line with the findings of Launay et al. (2006), who studied the shift in fiber type following a CTXinjection in the m. Soleus and in the m. Extensor digitorum longus (EDL) of 10-wk old mice(Launay et al. 2006). The authors reported that the early response in the EDL was characterized by the presence of type IIx and type IIb fibers 5 days after injection, while type IIa fibers firstly appeared after 14 days. As the EDL is also characterized by a fast fiber phenotype predominance, it is not surprising that the fiber type shift in this muscle was very similarly to the shift in the TA. Accordingly, in the slow $\mathrm{m}$. Soleus, type IIx and type IIb fibers were also the main fiber types five days following injection, while type IIa fibers were barely expressed. Seven days following CTX-injection, type IIa fibers became more apparent and at day 10, type I fibers appeared and their contribution increased until 28 days to become the mainly expressed fiber type. In agreement with our observations, these data confirm that in both 'slow' and 'fast' muscles, the formation of fasttwitch fibers precedes the slow-twitch fibers. However, in an 'intermediate' muscle, such as the m. Gastrocnemius, the formation of slow fibers preceded the fast fibers following a CTX-injection(Czerwinska et al. 2012). This discrepancy confirms that even within the same species and injury model, histological changes might profoundly differ amongst muscle types. Though, it cannot be excluded that different methodologies to assess the fiber type composition (immunofluorescence vs. MyHC gel electrophoresis) might also have contributed to the dissimilarities among these studies.

Besides the relative and absolute fiber type change during regeneration, we also related the regional appearances of specific fiber types to the muscle area integrity. At d2, the damaged muscle regions, characterized by mononucleated cell infiltration, were low in type IIa fibers, suggesting that these fibers are the most vulnerable to CTX-injection. At d5, it was apparent that muscle fibers with a centralized nucleus were almost exclusively type IIb and unstained fibers. Furthermore, the mimimum Feret diameter of type IIb and unstained fibers at d5 was significantly lower in CTX than in PBS, so it is likely that these fibers were newly formed between $\mathrm{d} 2$ and $\mathrm{d} 5$. One might suggest that unstained fibers at $\mathrm{d} 5$ represent immature embryogenic fibers. Indeed, classic work from Schiaffino showed the embryogenic and neonatal MHC isoforms are reexpressed after a denervation injury in rat skeletal muscle(Schiaffino et al. 1988). Also during recovery from a myotoxin injection in the rat $\mathrm{m}$. Soleus, embryogenic and neonatal fibers appear very early (d3-d4) in the regeneration process(D'Albis et al. 1988; Jerkovic et al. 1997). Given their increased number and decreased size at d5, it is thus very likely that unstained fibers at $\mathrm{d} 5$ represent indeed immature embryogenic fibers, rather than fibers that lost their mature MyHC isoform due to the injury. At d12, besides a persistent loss of type IIa fibers (0.5-fold $v s$. PBS), the relative fiber type composition of CTX was largely recovered (resembling the PBS condition). This is in agreement with d'Albis et al., who concluded that two weeks upon CTX injury in rats, the muscle MHC pattern was very similar to controls(D'Albis et al. 1988). However, fiber sizes observed in our study remained small in PBS vs. CTX, indicating that a time frame $>12$ days is necessary for full fiber recovery towards a healthy muscle phenotype. To our knowledge, this is the first study that relates the fiber type shift to regional changes in the muscle cross-sectional area's integrity.

Muscle stress response - In their response upon injury, molecular targets exhibit differential expression patterns, reflecting their unique functioning during the regeneration process. CTX-injection in skeletal muscle tissue evokes numerous physiological changes, which might each (in)dependently activate stress responses. Both the absence and an exuberance of stress/inflammatory signaling upon injury negatively impact the structural and the functional recovery of skeletal muscle tissue(Bohnert et al. 2018; Yang and Hu 2018). This implies that a good understanding of the stress response is important for the development of strategies to improve recovery. When therapies for muscle regeneration are studied, changes in the expression of stress markers are often used to interpret the therapy-efficacy and to understand the underlying mechanism(s). Therefore, the selection of particular 'stress targets' is important, as their expression patterns strongly differ and might result in different interpretations.

BiP, a chaperone located in the lumen of the endoplasmic reticulum (ER), is a member of the heat shock proteins (HSPs). It binds newly-synthesized proteins but also marks proteins for the degradation via the ubiquitin-proteasome pathway, especially when unfolded proteins accumulate in the ER(Gething 1999). BiP is highly expressed in response to various physiological stresses, including acute muscle injuries(Senf 2013), and more specifically to changes in intracellular calcium concentrations, glucose starvation or heat(Waser et al. 1997). Since it is known that CTX-injection induces an influx of calcium in muscle tissue(Harvey et al. 1982; Ownby et al. 1993), it is likely that this contributed to the acutely elevated BiP expression at d2. In their microarray approach, Yan et al. observed that Hsp70 and Hsp86 expression levels were highly upregulated early upon CTX injury (d1-d3) and downregulated at a later phase (d14)(Yan et al. 2003). Accordingly, Senf et al. found that the Hspa1a (coding for HSP70) mRNA expression was significantly elevated 1 and 16 days following CTX-injection, while no increase was observed at day 4, indicating a biphasic response of HSP70 gene expression upon injury(Senf et al. 2013). It remains unclear to which purpose the second peak serves. Our data also revealed an acutely 
elevated BiP protein expression at $\mathrm{d} 2$ and $\mathrm{d} 5$, but no second peak at $\mathrm{d} 12$. Interestingly, mitochondrial proteomic analysis revealed a downregulated expression of HSPd1 and HSPa9 (stress protein similar to Hsp70), with 3.3-fold and 2.5-fold, respectively, 3 days following a CTX injury(Ramadasan-Nair et al. 2014). However, one would expect that the expression of those proteins is increased to properly assemble unfolded polypeptides generated under stress conditions, i.e. tissue damage, in the mitochondrial matrix.

The BiP protein expression, which peaked at $\mathrm{d} 2$, preceded the upregulation of $\mathrm{p}-\mathrm{mTOR}$ at $\mathrm{d} 5$. It is therefore more likely that the BiP levels reflect their function to chaperone damaged proteins for ubiquitine-proteasome mediated degradation(Selsby et al. 2007), rather than their function to chaperone newly formed proteins, since this would imply an earlier activation of mTORC1 signaling, the central regulator of protein synthesis. Accordingly, Kojima et al. suggested that an elevated HSP72 protein expression was unrelated to protein synthesis in the necrosis-regenerating process following CTX-injection(Kojima et al. 2007). Alternatively, increased BiP levels might also protect against muscle damage, e.g. through its antioxidant capacity(Yan et al. 2002), and thereby promoting muscle regeneration and recovery(McArdle et al. 2004; Miyabara et al. 2006; Selsby et al. 2007). In mice, genetically-induced HSP overexpression(McArdle et al. 2004) and heat-induced HSP upregulation(Selsby et al. 2007) were effective to attenuate the loss in muscle strength induced by eccentric contractions(McArdle et al. 2004) and to improve muscle reloading following immobilization-induced atrophy(Selsby et al. 2007) by a HSP-induced inhibition of the oxidative damage(Selsby et al. 2007).

The MAPK family is involved in directing the cellular response to diverse stress stimuli, including osmotic stress, mitogens, heat and proinflammatory cytokines(Roux and Blenis 2004). ERK1, ERK2 and p38 MAPK are engaged in both the adaptation to environmental stress, and in the regulation of cell proliferation, cell cycle progression, cell division and differentiation. Although they belong to the same family, the patterns of expression differed between p-ERK1/2 and p-p38 MAPK, suggesting a distinct role during muscle regeneration. The peak expression of p-ERK1/2 five days following injury was also observed in rats 5 days upon notexin injection in the $\mathrm{m}$. Soleus $(\sim+330 \% v s$. intact muscle)(Richard-Bulteau et al. 2008). However, p-ERK1/2 was still increased 14 days following notexin injection although less pronounced ( + $60 \%)$, whereas we observed no difference at d12 between CTX and PBS. Similar to our findings, p-p38 MAPK was not increased 5 days upon notexin injection, but at d14, notexin increased p-p38 MAPK expression vs. the intact control, while we observed a decreased expression 12 days following CTX injection. Differences in expression patterns of the MAPKs can be attributed to a different species (rat $v s$. mice), muscle (Soleus vs. TA) and injury model (notexin $v s$. CTX).

MAPK expression might be the result of the proinflammatory milieu in skeletal muscle upon CTX injury. However, if this were the case, MAPK expression would peak at $\mathrm{d} 2$, as mononuclear cell infiltration and muscle damage was more pronounced at this time point (Fig. 1), and not at d5. Alternatively, the higher expression might have been partly related to a shift in fiber type composition, i.e. due to the increase in IIb fibers. In C57BL/6 mice, p-ERK1/2 expression was shown to be $\sim 2$-fold greater in the fast TA and Gastrocnemius than in the slow Soleus muscle(Shi et al. 2007, 2008). P-p38 was not differentially expressed according to the muscle type(Shi et al. 2008). Since both ERK1/2 and p38 are involved in myoblast proliferation and differentiation(Jones et al. 2001; Keren et al. 2006; Li and Johnson 2006; Cho et al. 2007; Yokoyama et al. 2007; Segalés et al. 2016), it is hard to distinguish their distinct effects on the skeletal muscle regeneration process based on their expression.

FOXO transcription factors are involved in the muscle energy homeostasis and are key regulators of the protein breakdown through modulation of the ubiquitin-proteasome and autophagy-lysosomal pathways(Sanchez and Candau 2014). FOXO is deactivated when phosphorylated and translocates to the nucleus upon dephosphorylation to exert its function as a transcription factor and to activate atrogenes, such as MuRF-1 and MaFbx(Brocca et al. 2017). Therefore, the decreased phosphorylated protein expression might reflect the increased activation of protein breakdown pathways. This is in accordance with the increased BiP expression, which marks proteins for degradation. Upon muscle damage, protein breakdown, partly regulated via FOXO signaling, is necessary for the recycling of amino acids, which are used for tissue rebuilding(Bröer and Bröer 2017). At d12, phosphorylated FOXO is no longer significantly different between PBS and CTX, which indicates that catabolic processes are now decreased compared to the early phases in muscle regeneration.

Muscle anabolic signaling - Besides the upregulation of a muscle stress response, muscle regeneration is also characterized by an anabolic response(Ge et al. 2009). mTORC1 signaling, which is the key regulator of protein synthesis, plays a crucial role in tissue regeneration. Surprisingly, however, there are no data available on the expression pattern of this pathway during regeneration. Upstream of mTOR, Akt can be activated by stimuli such as insulin, growth factors, calcium and cAMP(Filippa et al. 1999). As mentioned above, CTX-induced muscle injury is characterized by the influx of calcium, which might have contributed to the upregulation of the Akt-mTOR axis. In the present study, the expression of the upstream mTORC1 intermediates, e.g. p-Akt and p-mTOR, peaked 5d following CTX injury compared to PBS. Accordingly, IGF-2 gene expression also peaked five days following CTX injury(Yan et al. 2003). It was earlier shown that mTORC1 signaling regulates the IGF-2 expression during skeletal muscle myogenesis, both in vitro(Erbay et al. 2003) and in vivo(Ge et al. 2009). It is remarkable that at $\mathrm{d} 2$, the downstream mediators p-S6K1 and p-rpS6 exhibited a relatively 
higher protein expression $(+740 \%$ and $+260 \%$ vs. PBS $)$ than the upstream proteins p-Akt and p-mTORC1 $(+180 \%$ and $+40 \%$ vs. PBS). It cannot be excluded that we might have missed an initial calcium-induced transient peak (before $\mathrm{d} 2$ ) in p-Akt and p-mTOR expression upon CTX-injection, leading to an upregulation of the downstream proteins observed at d5. However, it is also possible that the calcium might have directly activated S6K1, independent of Akt(Graves et al. 1997; Conus et al. 1998; Gulati et al. 2008).

It can be questioned whether changes in the expression of the markers of muscle anabolism, i.e. mTORC1 signaling, reflect the protein synthesis or are rather indicative of muscle remodeling. Only a few studies looked at the real protein dynamics in muscle injury models. When nerve transfection/denervation was applied as a muscle injury model in rats, the muscle protein breakdown was acutely upregulated, while the protein synthesis was elevated only 7-10 days following injury(Goldspink 1976, 1978; Goldspink et al. 1983). Lately, it was confirmed that muscle protein synthesis was elevated 14-28 days upon nerve constriction in rats, despite a loss of $\sim 50 \%$ in muscle tissue (Langer et al. 2018). It should be noted, though, that the muscle atrophy process differs following a CTX-injection or following denervation. Still, these observations, which advocate that the mTORC1 activation reflects muscle remodeling rather than protein synthesis, correspond with our data as the upregulation of p-mTORC1 at $\mathrm{d} 5$ occurred with TA atrophy, i.e. a decreased muscle mass and a decreased IIb fiber size. It was therefore suggested that elevations in muscle protein synthesis, and thus in mTORC1 signaling, upon injury are indicative of muscle remodeling (e.g. myoblast proliferation, differentiation and fusion) rather than muscle growth per se(Ochala et al. 2011; Langer et al. 2018).

Integrative muscle signaling - Our data reveal that CTX severely impacts muscle histology and molecular signaling. To better understand the regeneration process, the potential physiological meaning(s) of the kinetics of each target should be interpreted in relation to the others. This might help researchers in their selection of targets and in data interpretation when applying a CTX injury model. In the present study, CTX activated two main pathways involved in the muscle regeneration, i.e. mTORC1 and ERK1/2 signaling. Both pathways exhibited similar expression patterns, i.e. a peak at d5 and a return towards baseline levels at d12. It was earlier suggested that the activation of mTORC1 signaling might be partially modulated by MAPK signaling(Roux and Blenis 2004; Miyazaki and Takemasa 2017). However, it is also possible that a same upstream mediator is at work for both targets. Given the elevated intracellular calcium concentrations upon muscle injury, it is possible that protein kinase $\mathrm{C}$ (PKC), induced by calcium, stimulated both mTORC1 signaling(Iijima et al. 2002; Fan et al. 2009; Miyazaki 2013; Osta et al. 2014) and ERK1/2(Cho et al. 2005; Tsao et al. 2013). In cardiac muscle cells, it was shown that mTORC1 activation was regulated through PKCs(Moschella et al. 2007). It remains to be established, whether this interaction also takes place in skeletal muscle cells. Furthermore, it should be noted that the activation of the analyzed stress markers and anabolic markers do not all necessarily result from the muscle fibers themselves, but might also be (partly) explained by immune and stromal cells, as their infiltration and proliferation takes places during the regeneration process. Indeed, the ERK pathway plays a central role in immune cells(Krzyzowska et al. 2010). Therefore, changes in immune cell number or phenotype during regeneration might also contribute to changes in pathway expression.

In conclusion, this is the first study that describes the molecular changes of pathways which play a central role during muscle regeneration following CTX-injection. Our study shows that the CTX-induced injury resulted in a rapid loss in type IIa muscle fibers, which was compensated by higher levels of type IIb fibers. Furthermore, CTX induced an early atrophic response, characterized by an acutely elevated increase in $\mathrm{BiP}$ and decrease in p-FoXO1/3a expression, eventually resulting in a decreased muscle mass and increase in type IIb muscle fibers. The activation of the mTOR and ERK1/2 pathway are very likely to be engaged in modulating cell proliferation and differentiation, and cell reconstruction via protein synthesis. The time-dependent and differential behavior of the targets indicate that researchers should carefully consider the selection of time point(s) upon injury and of specific targets to estimate the rate of injury/stress or recovery. 
Bohnert KR, Mcmillan JD, Kumar A (2018) Emerging roles of ER stress and unfolded protein response pathways in skeletal muscle health and disease. J Cell Physiol 233:67-78. https://doi.org/10.1002/jcp.25852

Brocca L, Toniolo L, Reggiani C, et al (2017) FoxO-dependent atrogenes vary among catabolic conditions and play a key role in muscle atrophy induced by hindlimb suspension. J Physiol 595:1143-1158. https://doi.org/10.1113/JP273097

Bröer S, Bröer A (2017) Amino acid homeostasis and signalling in mammalian cells and organisms. Biochem J 474:1935-1963. https://doi.org/10.1042/BCJ20160822

Cho H, Choi SH, Hwang K, et al (2005) Molecules and The Src / PLC / PKC / MEK / ERK Signaling Pathway Is Involved in Aortic Smooth Muscle Cell Proliferation induced by Glycated LDL. Mol Cells 19:60-66

Cho Y, Yao K, Bode AM, et al (2007) RSK2 Mediates Muscle Cell Differentiation through Regulation of NFAT3. J Biol Chem 282:8380-8392. https://doi.org/10.1074/jbc.M611322200

Conus NM, Hemmings BA, Pearson RB (1998) Differential Regulation by Calcium Reveals Distinct Signaling

Couteaux R, Mira J-C, D'Albis A (1988) Regeneration of muscles after cardiotoxin injury I. Cytological aspects. Biol Cell 62:171-182

Czerwinska AM, Streminska W, Ciemerych MA, Grabowska I (2012) Mouse gastrocnemius muscle regeneration after mechanical or cardiotoxin injury. Folia Histochem Cytobiol 50:144-153. https://doi.org/10.5603/FHC.2012.0021

D'Albis A, Couteaux R, Janmot C, et al (1988) Regeneration after cardiotoxin injury of innervated and denervated slow and fast muscles of mammals. Eur J Biochem 174:103-110

Erbay E, Park I, Nuzzi PD, et al (2003) IGF-II transcription in skeletal myogenesis is controlled by mTOR and nutrients. J Cell Biol 163:931-936. https://doi.org/10.1083/jcb.200307158

Fan Q, Cheng C, Knight ZA, et al (2009) EGFR Signals to mTOR Through PKC and Independently of Akt in Glioma. Sci Sign 2:ra4

Filippa N, Sable CL, Filloux C, et al (1999) Mechanism of Protein Kinase B Activation by Cyclic AMP-Dependent Protein Kinase. Mol Cell Biol 19:4989-5000

Fink E, Fortin D, Serrurier B, et al (2003) Recovery of contractile and metabolic phenotypes in regenerating slow muscle after notexin-induced or crush injury. J Muscle Res Cell Motil 24:421-429. https://doi.org/10.1023/A:1027387501614

Ge Y, Wu A, Warnes C, et al (2009) mTOR regulates skeletal muscle regeneration in vivo through kinase-dependent and kinase-independent mechanisms. Am J Physiol Cell Physiol 297:1434-1444. https://doi.org/10.1152/ajpcell.00248.2009.

Gething M (1999) Role and regulation of the ER chaperone BiP. Semin Cell Dev Biol 10:465-472

Goetsch SC, Hawke TJ, Gallardo TD, et al (2003) Transcriptional profiling and regulation of the extracellular matrix during muscle regeneration. Physiol Genomics 14:261-271

Goldspink DF (1978) Changes in the Size and Protein Turnover of the Soleus Muscle in Response to Immobilization or Denervation. Biochem Soc Trans 6:1014-1017

Goldspink DF, Garlickt PJ, Mcnurlanti MA (1983) Protein turnover measured in vivo and in vitro in muscles undergoing 
compensatory growth and subsequent denervation atrophy. Biochem J 210:89-98

Graves LM, He Y, Lambert J, et al (1997) An Intracellular Calcium Signal Activates p70 but Not p90 Ribosomal S6 Kinase in Liver Epithelial Cells. J Biol Chem 272:1920-1928

Gulati P, Gaspers LD, Dann SG, et al (2008) Article Amino Acids Activate mTOR Complex 1 via Ca2+/CaM Signaling to hVps34. Cell Met 4:456-465. https://doi.org/10.1016/j.cmet.2008.03.002

Harris JB (2003) Myotoxic phospholipases A2and the regeneration of skeletal muscles. Toxicon 42:933-945. https://doi.org/10.1016/j.toxicon.2003.11.011

Harvey A, Marshall R, Karlsson E (1982) Effects of purified cardiotoxins from the Thailand cobra (Naja naja siamensis) on isolated skeletal and cardiac muscle preparations. Toxicon 20:379-396

Iijima Y, Laser M, Shiraishi H, et al (2002) c-Raf / MEK / ERK Pathway Controls Protein Kinase C-mediated p70S6K Activation in Adult Cardiac Muscle Cells. J Biol Chem 277:23065-23075. https://doi.org/10.1074/jbc.M200328200

Järvinen TAH, Järvinen TLN, Kääriäinen M, et al (2005) Muscle Injuries: Biology and Treatment. Am J Sport Med 33:745-764. https://doi.org/10.1177/0363546505274714

Jerkovic R, Argentini C, Serrano-sanchez A, et al (1997) Early Myosin Switching Induced by Nerve Activity in Regenerating Slow Skeletal Muscle. Cell Struct Funct 22:147-153

Jones NC, Fedorov Y V., Rosenthal RS, Olwin BB (2001) ERK1/2 is required for myoblast proliferation but is dispensable for muscle gene expression and cell fusion. J Cell Physiol 186:104-115. https://doi.org/10.1002/10974652(200101)186:1<104::AID-JCP1015>3.0.CO;2-0

Keren A, Tamir Y, Bengal E (2006) The p38 MAPK signaling pathway: A major regulator of skeletal muscle development. Mol Cell Endocrinol 252:224-230. https://doi.org/10.1016/j.mce.2006.03.017

Kojima A, Goto K, Morioka S, et al (2007) Heat stress facilitates the regeneration of injured skeletal muscle. J Orthop Sci 12:74-82. https://doi.org/10.1007/s00776-006-1083-0

Krzyzowska M, Swiatek W, Fijalkowska B, et al (2010) The role of map kinases in immune response. Adv Cell Biol 2:125-138. https://doi.org/10.2478/v10052-010-0007-5

411 Langer HT, Senden JMG, Gijsen AP, et al (2018) Muscle Atrophy Due to Nerve Damage Is Accompanied by Elevated

412 Myofibrillar Protein Synthesis Rates. Front Physiol 9:1220. https://doi.org/10.3389/fphys.2018.01220

Launay T, Noirez P, Butler-browne G, et al (2006) Mechanisms of Tissue Repair Expression of slow myosin heavy chain during muscle regeneration is not always dependent on muscle innervation and calcineurin phosphatase activity. Am J Physiol Regul Integr Comp Physiol 290:1508-1514. https://doi.org/10.1152/ajpregu.00486.2005.

Lefaucheur JP, Bille ASI (1995) The cellular events of injured muscle regeneration depend on the nature of the injury. Neuromuscul Disord 5:501-509

Li J, Johnson SE (2006) ERK2 is required for efficient terminal differentiation of skeletal myoblasts. Biochem Biophys Res Commun 345:1425-1433. https://doi.org/10.1016/j.bbrc.2006.05.051

Mahdy M (2018) Glycerol-induced injury as a new model of muscle regeneration. Cell Tissue Res 374:233-241. https://doi.org/10.1007/s00441-018-2846-6

Mahdy MAA, Lei HY, Wakamatsu J-I, et al (2015) Comparative study of muscle regeneration following cardiotoxin and glycerol injury. Ann Anat 202:18-27. https://doi.org/10.1016/j.aanat.2015.07.002 muscle damage and age-related muscle dysfunction. FASEB J 18:355-357 
Miyabara EH, Martin JL, Griffin TM, et al (2006) Overexpression of inducible 70-kDa heat shock protein in mouse attenuates skeletal muscle damage induced by cryolesioning. Am J Physiol Cell Physiol 290:1128-1138. https://doi.org/10.1152/ajpcell.00399.2005.

Miyazaki M (2013) PKC-dependent regulation of mTOR activity is mediated through TSC2/Rheb signaling in C2C12 myoblasts. In: Proceedings of The Physiological Society. p PCA270

Miyazaki M, Takemasa T (2017) TSC2 / Rheb signaling mediates ERK-dependent regulation of mTORC1 activity in C2C12 myoblasts. FEBS Open Bio 7:424-433. https://doi.org/10.1002/2211-5463.12195

Moschella PC, Rao VU, Mcdermott PJ, Kuppuswamy D (2007) Regulation of mTOR and S6K1 activation by the nPKC isoforms , PKC $\varepsilon$ and PKC $\delta$, in adult cardiac muscle cells. J Mol Cell Cardiol 43:754-766. https://doi.org/10.1016/j.yjmcc.2007.09.015

Ochala J, Gustafson A, Diez ML, et al (2011) Preferential skeletal muscle myosin loss in response to mechanical silencing in a novel rat intensive care unit model : underlying mechanisms. J Phsyiol 589:2007-2026. https://doi.org/10.1113/jphysiol.2010.202044

Osta M El, Liu M, Adada M, et al (2014) Sustained PKCbII activity confers oncogenic properties in a phospholipase Dand mTOR-dependent manner. FASEB J 18:495-505. https://doi.org/10.1096/fj.13-230557

Ownby CL, Fletcher JE, Colberg TR (1993) Cardiotoxin 1 from cobra (Naja naja atra) venom causes necrosis of skeletal muscle in vivo. Toxicon 31:697-709. https://doi.org/10.1016/0041-0101(93)90376-T

Pessina P, Cabrera D, Morales MG, et al (2014) Novel and optimized strategies for inducing fibrosis in vivo: Focus on Duchenne Muscular Dystrophy. Skelet Muscle 4:1-17. https://doi.org/10.1186/2044-5040-4-7

Ramadasan-Nair R, Gayathri N, Mishra S, et al (2014) Mitochondrial Alterations and Oxidative Stress in an Acute Transient Mouse Model of Muscle Degeneration. J Biol Chem 289:485-509. https://doi.org/10.1074/jbc.M113.493270

Richard-Bulteau H, Serrurier B, Crassous B, et al (2008) Recovery of skeletal muscle mass after extensive injury: Positive effects of increased contractile activity. Am J Physiol - Cell Physiol 294:467-476. https://doi.org/10.1152/ajpcell.00355.2007

Roux PP, Blenis J (2004) ERK and p38 MAPK-Activated Protein Kinases : a Family of Protein Kinases with Diverse Biological Functions. Microbiol Mol Biol Rev 68:320-344. https://doi.org/10.1128/MMBR.68.2.320

Sanchez AMJ, Candau RB (2014) FoxO transcription factors : their roles in the maintenance of skeletal muscle homeostasis. Cell Mol Life Sci 71:1657-1671. https://doi.org/10.1007/s00018-013-1513-z

Schiaffino S, Gorza L, Pitton G, et al (1988) Embryonic and neonatal myosin heavy chain in denervated and paralyzed rat skeletal muscle. Dev Biol 127:1-11. https://doi.org/10.1016/0012-1606(88)90183-2

Segalés J, Perdiguero E, Muñoz-cánoves P (2016) Regulation of Muscle Stem Cell Functions : A Focus on the p38 MAPK Signaling Pathway. Front Cell Dev Biol 4:91. https://doi.org/10.3389/fcell.2016.00091

Selsby JT, Rother S, Tsuda S, et al (2007) Intermittent hyperthermia enhances skeletal muscle regrowth and attenuates oxidative damage following reloading. J Appl Physiol 102:1702-1707. https://doi.org/10.1152/japplphysiol.00722.2006.

Senf SM (2013) Skeletal muscle heat shock protein 70: diverse functions and therapeutic potential for wasting disorders. Front Physiol 4:330. https://doi.org/10.3389/fphys.2013.00330

Senf SM, Howard TM, Ahn B, et al (2013) Loss of the Inducible Hsp70 Delays the Inflammatory Response to Skeletal Muscle Injury and Severely Impairs Muscle Regeneration. PLoS One 8:e62687. https://doi.org/10.1371/journal.pone.0062687 
Shi H, Scheffler JM, Pleitner JM, et al (2008) Modulation of skeletal muscle fiber type by mitogen- activated protein kinase signaling. FASEB J 22:2990-3000. https://doi.org/10.1096/fj.07-097600

Shi H, Zeng C, Ricome A, et al (2007) Extracellular signal-regulated kinase pathway is differentially involved in betaagonist-induced hypertrophy in slow and fast muscles. Am J Physiol Cell Physiol 292:1681-1689. https://doi.org/10.1152/ajpcell.00466.2006.

Sunitha B, Gayathri N, Kumar M, et al (2016) Muscle biopsies from human muscle diseases with myopathic pathology reveal common alterations in mitochondrial function. J Neurochem 138:174-191. https://doi.org/10.1111/jnc.13626

Tsao H, Chiu P, Sun SH (2013) PKC-dependent ERK phosphorylation is essential for P2X 7 receptor-mediated neuronal differentiation of neural progenitor cells. Cell Death Dis 4:e751. https://doi.org/10.1038/cddis.2013.274

Waser M, Mesaeli N, Spencer C, Michalak M (1997) Regulation of calreticulin expression by calcium. J Cell Biol 138:547-557

Yan L, Christians ES, Liu L, et al (2002) Mouse heat shock transcription factor 1 deficiency alters cardiac redox homeostasis and increases mitochondrial oxidative damage. EMBO J 21:5164-5172

Yan Z, Choi S, Liu X, et al (2003) Highly Coordinated Gene Regulation in Mouse Skeletal Muscle Regeneration. J Biol Chem 278:8826-8836. https://doi.org/10.1074/jbc.M209879200

Yang W, Hu P (2018) Skeletal muscle regeneration is modulated by inflammation. J Orthop Transl 13:25-32. https://doi.org/10.1016/j.jot.2018.01.002

Yokoyama T, Takano K, Yoshida A, et al (2007) DA-Raf1, a competent intrinsic dominant-negative antagonist of the Ras-ERK pathway, is required for myogenic differentiation. J Cell Physiol 177:781-793. https://doi.org/10.1083/jcb.200703195 
Fig. 1 Regeneration phases of TA muscle fibers. Histological sections stained with H\&E revealed muscle infiltration of mononucleated cells (red arrows) on day 2 (myolysis) and day 5 (early regeneration) following CTX-injection. At d5, damaged myofibers were replaced by small newly-formed myofibers with a centralized nucleus (white arrows). Twelve days upon CTX injury, myofibers with a centralized nucleus (white arrows) were enlarged and a larger contribution of myofibers with a nucleus in the periphery (green arrows) was observed. PBS-injection did not evoke muscle injury or regeneration, as healthy muscle fibers with peripheral nuclei were apparent 2,5 and 12 days upon injection. Scale bar $=$ $100 \mu \mathrm{m}$.

Fig. 2 Fibronectin (FN) in TA muscle during regeneration. Histological sections stained for FN and Hoechst (nuclei). Two days upon the cardiotoxin (CTX) injection, FN was highly expressed in damaged fibers, whereas FN was only expressed in the sarcolemma of PBS-injected fibers. At d5, the FN expression was still present in some but not all fibers in damaged areas. Infiltrated cells were present in the fibers that highly expressed FN (red arrows) but not in undamaged fibers that only expressed FN in the sarcolemma (white arrows). Twelve days following CTX-injection, the FN staining revealed that all fibers in regenerating areas expressed FN in the sarcolemma, but to a higher extent than the PBS-injected control. Scale bar $=100 \mu \mathrm{m}$.

Fig. 3 (n=7-8/condition): The amount of muscle type IIa (A), unstained (B) and type IIb fibers (C) during TA muscle regeneration expressed in absolute values. Data are expressed as mean \pm SEM. $* \mathrm{p}<0.05 v s$. PBS. Representative muscle sections immunostained for myosin heavy chain (MyHC) I (green), MyHC IIa (blue), Ilb (red) and laminin (green) (D). 505 Scale bar $=100 \mu \mathrm{m}$.

Fig. 4 (n=7-8/condition): The expression of different proteins involved in the regulation of cellular stress in the TA following CTX injury. The target molecules are binding immunoglobulin protein (BiP), phospho (p)-Forkhead box O3 (FOXO), p-p38 mitogen-activated protein kinases (p38MAPK), p-extracellular signal-regulated kinases (ERK). Data are expressed in mean \pm SEM. $* \mathrm{p}<0.05 v s$. PBS; $\uparrow \mathrm{p}<0.1$ vs. PBS.

510 Fig. 5 (n=7-8/condition): Expression of the mTOR signaling pathway in the TA following CTX injury. The target molecules 511 are (from upstream to downstream) phospho (p)-Akt, p-mammalian target of rapamycin (mTOR), p-ribosomal protein S6 512 kinase beta-1 (S6K1) and p-ribosomal protein S6 (rpS6). Data are expressed in mean \pm SEM. * p < $0.05 v s$. PBS. 
513 Body mass and m. Tibialis Anterior mass

\begin{tabular}{|l|c|c|c|c|c|c|}
\hline & \multicolumn{3}{|c|}{ PBS } & \multicolumn{3}{c|}{ CTX } \\
\cline { 2 - 7 } & d2 & d5 & d12 & d2 & d5 & d12 \\
\hline BM (start) (g) & $25.0 \pm 0.6$ & $25.8 \pm 0.7$ & $26.7 \pm 0.8$ & $25.0 \pm 0.2$ & $25.8 \pm 0.5$ & $26.5 \pm 0.5$ \\
\hline BM (end) (g) & $25.0 \pm 0.7$ & $25.4 \pm 0.4$ & $26.8 \pm 0.8$ & $24.1 \pm 0.2$ & $25.3 \pm 0.8$ & $27.2 \pm 0.5$ \\
\hline TA (mg) & $51.2 \pm 1.3$ & $50.6 \pm 1.0$ & $49.9 \pm 1.0$ & $49.1 \pm 1.8$ & $41.1 \pm 0.9 *$ & $50.0 \pm 1.9$ \\
\hline
\end{tabular}

514 Table 1 ( $\mathrm{n}=7-8 /$ condition). Data are presented as mean \pm SEM. BM: body mass; TA: tibialis anterior; $*$ P $<0.05$ vs. PBS.

515

516 Relative muscle fiber type composition and minimum Feret diameter

\begin{tabular}{|c|l|l|l|l|l|l|l|}
\hline & \multicolumn{2}{|l|}{ Day 2 } & \multicolumn{2}{l|}{ Day 5 } & \multicolumn{2}{l|}{ Day 12} \\
\hline $\begin{array}{c}\text { Fiber } \\
\text { Type }\end{array}$ & Parameter & PBS & CTX & PBS & CTX & PBS & CTX \\
\hline \multirow{2}{*}{ Type IIa } & Frequency (\%) & $16.0 \pm 3.1$ & $4.7 \pm 1.5 *$ & $17.2 \pm 2.7$ & $4.7 \pm 2.1 *$ & $19.4 \pm 4.2$ & $10.6 \pm 2.4$ \\
\cline { 2 - 8 } & $\begin{array}{l}\text { Min Feret } \\
\text { diam }(\mu \mathrm{m})\end{array}$ & $33.5 \pm 1.3$ & $28.3 \pm 2.1$ & $27.4 \pm 2.9$ & $29.8 \pm 1.3$ & $33.5 \pm 4.2$ & $27.0 \pm 2.2 \dagger$ \\
\hline \multirow{2}{*}{ Type IIx } & \begin{tabular}{l} 
Frequency $(\%)$ \\
\cline { 2 - 8 }
\end{tabular} & $32.0 \pm 5.6$ & $54.1 \pm 7.9 *$ & $28.6 \pm 2.7$ & $39.0 \pm 4.1$ & $24.5 \pm 5.2$ & $31.4 \pm 5.0$ \\
\hline Miam Feret $(\mu \mathrm{m})$ & $36.0 \pm 1.3$ & $33.6 \pm 1.7$ & $31.3 \pm 2.3$ & $21.9 \pm 1.3 *$ & $36.5 \pm 2.2$ & $28.4 \pm 3.4 *$ \\
\hline \multirow{2}{*}{ Type IIb } & \begin{tabular}{l} 
Frequency $(\%)$ \\
\cline { 2 - 8 }
\end{tabular} & $52.0 \pm 4.1$ & $41.2 \pm 6.8 \dagger$ & $54.2 \pm 1.8$ & $56.2 \pm 2.6$ & $56.1 \pm 4.0$ & $57.9 \pm 3.9$ \\
\hline
\end{tabular}

517 Table 2 ( $\mathrm{n}=6-8 /$ condition). Data are presented as mean \pm SEM $(\mathrm{n}=8)$. Min Feret diam: minimum Feret diameter; $* \mathrm{p}<0.05$

$518 v s$. PBS; $\dagger \mathrm{p}$ between 0.05-0.1 vs. PBS. 